\title{
Regulating the Nuclear Reactor through Changes of the Fraction of Delayed Neutrons: Theoretical Probabilities
}

\author{
Dmitry V. Filippov ${ }^{1}$, Leonid I. Urutskoev ${ }^{1}$, Valery I. Rachkov ${ }^{2}$, Olga I. Gadzaova ${ }^{1}$, \\ Larion A. Lebedev ${ }^{3}$ \\ ${ }^{1}$ Moscow State University of Printing Arts, Russia \\ ${ }^{2}$ State Atomic Energy Corporation "Rosatom", Moscow, Russia \\ ${ }^{3}$ State Research and Development Center for Expertise of Projects and Technologies, Moscow, Russia \\ E-mail: \{filippov-atom,urleon\}@ya.ru \\ Received August 1, 2010; revised October 19, 2010; accepted October 17, 2010
}

\begin{abstract}
In recent years a significant number of both theoretical and experimental works devoted to the influence of external electromagnetic fields and ionization on the probability of beta decays have been published. The present work investigates the feasibility of using this physical effect as the main mechanism for controlling the reactor. In this paper a system of equations is written and studied that allows one to describe the work of a nuclear reactor in the case where the probability of beta decay and, therefore, the fraction of delayed neutrons is a function of time. It is shown that in the case of a constant fraction of delayed neutrons, the proposed system of equations is identical to the known system. As can be seen from analysis of a solution of the new system of equations for the proposed method of reactor control, acceleration by instantaneous neutrons is impossible even theoretically.
\end{abstract}

Keywords: Fraction of Delayed Neutrons, Bound-State Beta-Decay, Kinetic Equation

\section{Introduction}

During recent years, our understanding has been that the impact of atomic electron shell disturbances on the periods of nuclear decay caused by weak electromagnetic interactions could be quite significant. For example, the ${ }^{163} \mathrm{Dy},{ }^{193} \mathrm{Ir},{ }^{205} \mathrm{Tl}$ nuclei, which are absolutely stable in neutral atoms, become $\beta$-active under full atom ionization [1], while complete ionization of ${ }^{187} \mathrm{Re}$ increases the possibility of $\beta$-decay by a factor of $10^{9}$ [2]. The probabilities for $\beta$-decay increase not only under ionization but when an atom is exposed to a superstrong magnetic field [3]. Since the physical mechanism of the production of delayed neutrons (DN) from nuclear emitters is directly connected to $\beta$-decay processes, the question was reasonably raised [4] of whether it is possible to change the DN fraction. [5,6], it was proved quite convincingly that the DN fraction definitely increased under ionization of atoms.

The appearance of DN during uranium fission is a basic physical effect making it possible to create a nuclear reactor and underlying the operation control for reactors of any type. Particularly significant in this respect is the influence of DN on the behavior of the reactor with circulating fuel [7]. Although at present the possibility of changing the DN fraction through external impacts causes no doubts when describing the atomic reactor kinetics, it is nevertheless considered that the DN fraction of each particular nuclear emitter does not depend on external conditions. This discrepancy can be explained by the fact that the theoretical foundations of reactor operation had been developed long before they received reliable experimental data witnessing the influence of external physical impacts on the probability of nuclear processes that involve weak interactions. At present, when describing the reactor kinetics, consideration is taken only of the variation of the average DN fraction during the reactor process due to a different chemical composition of the active zone. This work is aimed at qualitative analysis of whether the method based on changing the DN fraction could form (at least theoretically) the basis for regulating the said nuclear reactor.

The classical equations of reactor kinetics [7] were basically written under invariable DN fraction conditions. Hence, analyzing them in the framework of the variable DN fraction would not be quite correct. This work for- 
mulates the reactor kinetics equations as based on the whole quantity of the DN nuclear emitters (including those whose decays do not result in forming neutrons). These equations are analyzed in the case of a change in the fraction of DN. It is shown that when using external impacts (e.g. superpowerful magnetic field) to change the fraction of DN, then theoretically, in a way it is possible to regulate the power of the reactor.

\section{Effect of Bound-State Beta-Decay on the Fraction of Delayed Neutrons}

A theory of bound-state $\beta^{-}$-decay, in which the beta electron does not leave the atom but occupies a free orbit, was constructed in [8-11]. The ratios of the decay constants (ratios of the $\beta^{-}$-decay probabilities) for transitions to bound and free states ( $\lambda_{b}$ and $\lambda_{c}$, respectively) were calculated in [9-11]. For $\beta^{-}$-decays of low energy in fully ionized heavy atoms, the ratio $\lambda_{b} / \lambda_{c}$ may be as large as $10^{3}-10^{4}$. Thus, we see that, in the presence of free electron orbits, the probability of the $\beta^{-}$-decay of nuclei may increase by three orders of magnitude or more. The theory of bound-state $\beta^{-}$-decay was experimentally confirmed in $[1,2]$.

However, the allowance for bound-state beta-decay may prove to be of importance not only in the case where the nucleus involved has an anomalously low boundary energy for beta decay, ${ }^{187} \mathrm{Re}$, for example, possesses this property [2]; but also in the case where the decay process being considered proceeds via various channels, including those of decay to highly excited levels of the daughter nucleus. In the former case, the decay half-life changes, while, in the latter case, the change in the decay half-life is small, but the ratio of the intensities of decays through different channels may undergo a substantial redistribution. This effect will lead to a change in the relationship between the intensities of the lines of gamma radiation from the daughter nucleus. If, in addition, an emitter of DN appears as the initial beta-decaying nucleus, the DN fraction will change.

The ratio of the probabilities of bound-state $\beta^{-}$-decay to free states can be calculated by a method similar to the classic method for calculating the ratio of the K-capture to the $\beta^{+}$-decay probability [12]. In the following, we will use the system of units in which $\hbar=\mathrm{c}=\mathrm{m}_{\mathrm{e}}=1$. For allowed beta decays, the decay constants for transitions to a bound and a free state are proportional to the same matrix elements and differ only in phase spaces of electron-neutrino final states. From $[11,12]$, it is well known that, for beta decay to a free state, the phase space is proportional to the integral Fermi function:

$$
f(Z, E)=\int_{1}^{E} F(Z, \varepsilon) \sqrt{\varepsilon^{2}-1}(E-\varepsilon)^{2} \varepsilon d \varepsilon,
$$

( $E$ is the beta-transition energy) and is the sum of all possible energies and momentum directions for the electron (neutrino). The Fermi function in (1) grows with energy faster than in proportion to $E^{2}$.

For bound-state beta-decay the neutrino spectrum is concentrated at a single energy value, since the energy of an electron moving along an orbit is fixed, and the phase space is determined by a possible arbitrary direction of the neutrino momentum. The phase space is then proportional to the product of the square of the neutrino momentum,

$$
p^{2}=\frac{1}{c^{2}}(E-1+\varepsilon)^{2}
$$

( $\varepsilon$ is the energy of the electron moving in the respective orbit) and the probability of the intersection of the free electron orbit and the nucleus involved. In turn, the intersection probability is proportional to $\left|\Psi_{e}(R)\right|^{2}$, where $\Psi_{e}(R)$ is the density of the electron wave function in the region occupied by the nucleus. For hydrogen-like orbit:

$$
\left|\Psi_{e}(0)\right|^{2} \sim \frac{1}{\pi}\left(\frac{\alpha Z}{m}\right)^{3},
$$

where $\alpha=1 / 137$ is the fine structure constant, $Z$ is the charge number of the nucleus and $m$ is the quantum number of state.

It follows that the appearance of a free electron orbit enhances the decay constant for the allowed beta transition of energy $E\left(\lambda_{0}\right)$ by the quantity $\Delta \lambda$,

$$
\frac{\Delta \lambda}{\lambda_{0}}=2 \pi^{2} \frac{\left|\Psi_{e}(R)\right|^{2}(E-1+\varepsilon)^{2}}{f(Z, E)} .
$$

Since, with increasing energy $E$, the Fermi function (1) grows faster than in proportion to $E^{2}$, the ratio $\Delta \lambda / \lambda_{0}$ decreases as the energy increases,

$$
\frac{\Delta \lambda}{\lambda_{0}} \infty \frac{1}{E^{\delta}}, \delta>0 .
$$

Thus, the lower the decay energy $E$, the more pronounced the increase in the decay constant because of decay to a bound state. It is noteworthy that the energy dependence of the decay constant takes the same form, irrespective of which electron orbit is free, since it is the factor $\left|\Psi_{e}(R)\right|^{2}$ that absorbs the effect of the distinction between the orbits. In order to derive the estimate in (3), we only employed the fact that the neutrinos accompanying bound-state beta-decay are monoenergetic.

In the particular case where a hydrogen-like orbit of the atom being considered is free (the case of a fully ionized atom), we find from (2) and (3) that

$$
\frac{\Delta \lambda}{\lambda_{0}} \sim 2 \pi \frac{(\alpha Z)^{3}(E-1+\varepsilon)^{2}}{f(Z, E)} .
$$


We note that the ratio $\Delta \lambda / \lambda_{0}$ is greater for forbidden than for allowed transitions since, in expression (5), the numerator features the beta-decay form factor at the maximum neutrino energy, while the denominator is equal to the same form factor averaged over all neutrino energies according to (1). For uniquely forbidden transitions, the ratio $\Delta \lambda / \lambda_{0}$ was considered in [11].

The fission of ${ }^{235} \mathrm{U}$ leads to the formation of a large number of fragments whose atomic weights range between $A=72$ and $A=160$. The mass and charge distributions of fission fragments have been well understood. The majority of the fragments are unstable neutron-rich nuclei [13]. Among these, about 50 nuclei are sources of $\mathrm{DN}$ and decay according to the scheme in the Figure 1.

The beta decay of the initial nucleus (DN emitter) through a channel characterized by lower beta-transition energies leads to the formation of an intermediate nucleus in an excited state. At an excitation energy above the neutron binding energy $\left(Q_{n}\right)$, the intermediate nucleus emits a neutron. Neutron emission from the intermediate nucleus is virtually prompt, the delay time being determined by the lifetime of the initial nucleus. We define: $\lambda$ as the constant of full decaying DN nuclear emitters and $\lambda_{n}$ as the constant of decaying DN nuclear emitters through the $\beta$-decay channel with the appearance of neutrons.

As can be seen from Figure 1, the $\beta$-decays of DN nuclear emitters resulting in the appearance of neutrons (taking place on the excited energy levels of intermediate nuclei) have significantly lesser decay energies than $\beta$-decays without the appearance of neutrons [13]. Hence, relative changing of $\lambda_{n}$ is much greater than relative changing of $\lambda$ (4) [3-6]. Let the following changing of $\beta$-decay take place:

$$
\lambda=\lambda_{0}+\Delta \lambda, \lambda_{n}=\lambda_{n 0}+\Delta \lambda_{n},
$$

then

$$
\frac{\Delta \lambda_{n}}{\lambda_{n 0}}>\frac{\Delta \lambda}{\lambda_{0}} .
$$

Let us define the fraction of DN $\eta$ in the following way:

$$
\eta=R \frac{\lambda_{n}}{\lambda},
$$

then the said changing of the $\beta$-decay probabilities results in a greater fraction of $\mathrm{DN} \eta=\eta_{0}+\Delta \eta\left(\eta_{0}\right.$ is the unexcited fraction of $\mathrm{DN}$ ):

$$
\frac{\Delta \eta}{\eta_{0}}=\frac{\lambda_{0}}{\lambda}\left(\frac{\Delta \lambda_{n}}{\lambda_{n 0}}-\frac{\Delta \lambda}{\lambda_{0}}\right)>0 .
$$

Therefore, the appearance of a free electron orbit in an atom that emits DN leads to an increase in the DN frac-

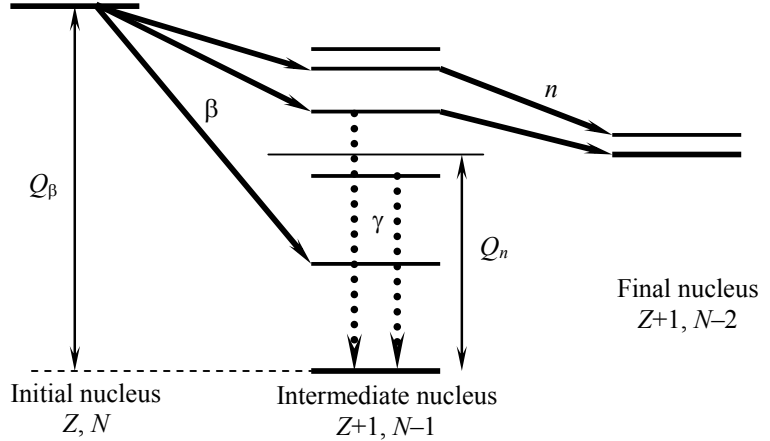

Figure 1. Scheme of the decay of a nucleus emitting delayed neutrons $\left(Q_{\beta}\right.$ is the maximum beta-decay energy, while $Q_{n}$ is the neutron binding energy in the intermediate nucleus).

tion. In $[5,6]$ the relative increase in the DN fraction of nuclei was calculated which originated from the fission of uranium and plutonium from the first three groups.

\section{Increase in the DN Fraction in a Superstrong Magnetic Field}

In [14-16] it is shown that upon placing of an atom in an external homogeneous constant strong magnetic field $H>H_{0}=\mathrm{cm}_{e}^{2} e^{3} \hbar^{-3} \approx 2.35 \times 10^{9} \mathrm{Gs}$, the properties of the atom qualitatively change. It follows from [14-16] that in such a field the density of electronic states in the nucleus increases and changes the ionization energy of the atom. In a superstrong magnetic field, the motion of atomic electrons in a plane perpendicular to the magnetic field occurs on the Landau levels. In the direction along the magnetic field the electron moves in one-dimensional Coulomb potential, averaged over the cross movement. In [3] the density of the electron orbit is calculated in the nucleus in a superstrong magnetic field. In contrast to (2) in the nucleus the density of electronic states with quantum number $m$ of longitudinal motion is

$$
\left|\Psi_{H e}(0)\right|^{2} \sim \frac{e H}{2 \pi}\left(\frac{\alpha Z}{m}\right) .
$$

Consequently, first, in superstrong magnetic field, the density of excited electronic states $(m>1)$ of the nucleus increases so much that bound-state $\beta$-decay of nuclei becomes significant not only for a fully ionized but also for a neutral atom (formally the sum $\Sigma m^{-1}$ diverges). Secondly, in the superstrong field, the density of unoccupied electronic states in the field of the nucleus (9) becomes proportional to the magnetic field $H$. Consequently, the probability of bound-state decay also becomes proportional to the strength of the magnetic field $H$. The probability of bound-state decay with complete ionization of an atom is limited to the size of the nuclear charge $Z$ (2), (3), and in a superstrong magnetic field, the 
probability of bound-state decay can indefinitely increase with a sufficiently large magnetic field (9).

From (3) and (8) subject to (9) it follows that upon placing of an atom with a nucleus of DN emitter into a superstrong magnetic field with strength $H$ such that

$$
e H \square \frac{\lambda}{\Delta \lambda_{I}}(\alpha Z)^{2},
$$

(where $\Delta \lambda_{I}<<\lambda$ is an increase of the total permanent $\beta$-decay due to the complete ionization of the atom), the increase in the fraction of $\mathrm{DN}$ is

$$
\Delta \eta_{H} \sim \Delta \eta_{I} \frac{e H}{(\alpha Z)^{2}} .
$$

Consequently, for a sufficiently large magnetic field $e H>(\alpha Z)^{2}$ the fraction of DN upon placing of an atom with a nuclear emitter of $\mathrm{DN}$ in a superstrong magnetic field grows more strongly than in the case of the full ionization of the atom.

The limiting increase in the fraction of $\mathrm{DN}$ in the magnetic field, the strength of which satisfies the inequality inverse to (10), is

$$
\left(\frac{\Delta \eta_{H}}{\eta}\right)_{\max } \sim \frac{\Delta \eta_{I}}{\eta} \frac{\lambda}{\Delta \lambda_{I}} \sim\left(\frac{E_{n}}{E_{\beta}}\right)^{2} \frac{f\left(Z, E_{\beta}\right)}{f\left(Z, E_{n}\right)},
$$

and does not depend on the magnetic field but is determined only by the ratio of the energy $E_{n}$ of $\beta$-decay to give a neutron and the energy $E_{\beta}$ of decay giving no neutron. For the first three groups of nuclear emitters of DN, which are the products of uranium fission, $(\Delta \eta / \eta)_{\max }>$ 25.

\section{Kinetic Equations Taking into Consideration Possible Changes in the Delayed Neutron Fraction}

The power of the reactor is proportional to the neutron density $n$. It is well known [7] that DN impacts on the reactor dynamics can be correctly described by kinetic equations in the framework of a homogeneous isotropic model. For thorough analysis of the reactor behavior under changing $\lambda$ ( $\beta$-decay constants) we will use the well accepted approximation of one efficient DN group. So let us first define the following variables: $n$ is the density of all neutrons in the active reactor zone; $Y$ is the density of all DN nuclear emitters in the active zone including those whose decay does not result in neutron formation. This value significantly differs from the density of nuclear emitters which is typically used in the classic kinetic equations where only nuclei decaying along with the appearance of the neutrons are taken into account.
Let us define $\chi$ as the prompt neutron cascade multiplication coefficient which is the ratio of the rate of the appearance of prompt neutrons to the rate of the absorption of all neutrons (the ratio of the number of instantaneous neutrons produced during a unit time in a unit volume to the number of all neutrons absorbed during the same time and in the same volume); $R$ as the ratio of the number of produced nuclear DN emitters to the number of produced prompt neutrons; $T$ as the effective life time of the generation of prompt neutrons such that $n T^{-1}$ is, by definition, the rate of the appearance of prompt neutrons (number of prompt neutrons produced during the unit time in the unit volume); $\lambda_{n}$ as the constant of decaying DN nuclear emitters through the $\beta$-decay channel with the appearance of neutrons, that is $\lambda_{n} Y$ is defined as the rate of the DN appearance (the number of DN produced during the unit time in the unit volume). It is well known that a small number of DN nuclear emitters produce more than one DN. We will take into account this property in $\lambda_{n}$. Finally we define $\lambda$ as the decay constant of DN nuclear emitters through all the $\beta$-decay channels under which the decay results in a nucleus not being DN emitter. It should be noted that $\beta$-decays resulting in the presence of a new nuclear emitter are usually not taken into account here: a nucleus that is a nuclei composition is well described by the $Y$ density factor. Taking into account the above notations, the equations for neutrons and nuclear emitters of DN are as follows:

$$
\begin{gathered}
\frac{d n}{d t}=\frac{n}{T}-\frac{1}{\chi} \frac{n}{T}+\lambda_{n} Y, \\
\frac{d Y}{d t}=R \frac{n}{T}-\lambda Y .
\end{gathered}
$$

The first term in the right-hand part of the first equation describes the appearance of instantaneous neutrons, the second term describes the absorption of neutrons and the third one characterizes the DN appearance. The first term in the right-hand part of the second equation describes the formation and the second term describes the disappearance of DN nuclear emitters.

Now let us suppose that some impact is imposed on the reactor active zone that slightly affects the process of enforced fission and absorption of neutrons but increases the probability of the $\beta$-decay (e.g. ionization or an intensive magnetic field). In such a case, $\lambda_{n}$ and $\lambda$ ratios are changed in Equations (13) while the other remain the same. Relative changing of $\lambda$ and $\lambda_{n}$ (6) results in a greater fraction of DN (8).

Further we will introduce the reactivity of nuclear reactor $\rho$ :

$$
\rho=\frac{\chi-1}{\chi}+\eta_{0}
$$


System (13) can be represented as

$$
\begin{aligned}
& \frac{d n}{d t}=\frac{\rho-\eta_{0}}{T} n+\lambda_{n} Y, \\
& \frac{d Y}{d t}=R \frac{n}{T}-\lambda Y .
\end{aligned}
$$

Now let us consider the behavior of the reactor which, being unexcited, operated in the stationary regime, that is $\rho=0$. We are primarily interested in solutions satisfying the following initial conditions:

$$
\frac{Y(0)}{R}=n(0) \frac{\eta_{0}}{R \lambda_{n 0} T}=\frac{n(0)}{\lambda_{0} T} .
$$

We will take into account the fact that changes $\Delta \lambda$ and $\Delta \lambda_{n}$ occur instantly (during the time $<<T$ ). Let us examine the behavior of the reactor with new time-independent $\lambda=\lambda_{0}+\Delta \lambda, \lambda_{n}=\lambda_{n 0}+\Delta \lambda_{n}$, and $\rho=0$. Then from (15), taking into account (8), we obtain the following equation:

$$
\frac{d^{2} n}{d t^{2}}+\frac{d n}{d t}\left[\frac{\eta_{0}}{T}+\lambda\right]-n \frac{\lambda \Delta \eta}{T}=0,
$$

which describes the reactor behavior when it deviates from equilibrium. Should such be reached under certain impact on the active zone (with excited values of $\lambda$ and $\eta$ ) then the deviation may occur in the case of the impact cut off (disturbance). Thus we can, generally speaking, consider $\Delta \lambda$ and $\Delta \eta$ in (17) both positive when putting on the impact and negative when taking it off.

Equation (17) describes an unstable singular saddleshaped point. It is not difficult to find solutions of the equation by solving its characteristic equation and determining the characteristic number $\kappa$ :

$$
\kappa=\frac{1}{2}\left(\frac{\eta_{0}}{T}+\lambda\right)\left[ \pm \sqrt{1+4 \frac{\lambda T \Delta \eta}{\left(\eta_{0}+\lambda T\right)^{2}}}-1\right] .
$$

In the approximation $\lambda T<<\eta_{0}$ we get

$$
\kappa_{+}=\lambda \frac{\Delta \eta}{\eta_{0}}, \kappa_{-}=-\frac{\eta_{0}}{T},
$$

and such solutions (19) are applicable to both cases of small $\Delta \eta<<\eta$ and greater $\Delta \eta>\eta$ excitations.

When including $(\Delta \eta>0)$ the said impact, the density of the $n$ neutrons will grow with the value $\kappa_{+}$(19) but then it will stop. Since the power in the reactor is proportional to the density of the neutrons $n$ [7] it becomes possible to change its power by exerting impacts on the active reactor zone by means of external fields. Now let us compare such a method of regulation with the classical one.

\section{Classical Kinetic Equations}

To come to the classical equations of kinetics, let us first define the text-book [7] value of $C$ as the DN density of nuclear emitters which have $\beta$-decay through the channels giving a neutron:

$$
\begin{gathered}
C=\frac{\lambda_{n}}{\lambda} Y=\frac{\beta Y}{R}, \\
\beta=\eta .
\end{gathered}
$$

In the classical approach to the problem, $\lambda_{n}, \lambda$, and hence, $\beta$ are constants, but in this case the substitution of (20) into (15) leads to the well known equations [7]

$$
\begin{gathered}
\frac{d n}{d t}=\frac{\rho-\beta}{T} n+\lambda C, \\
\frac{d C}{d t}=\frac{\beta n}{T}-\lambda C .
\end{gathered}
$$

Obviously, if $\beta$ is not a constant then the second equation in (21) should be different:

$$
\frac{d C}{d t}=\frac{\beta n}{T}-\lambda C+C \frac{d \ln \beta}{d t}
$$

Thus, the system of Equations (15) seems to be more general than (21) because the latter holds only for constant $\lambda_{n}, \lambda$, and $\beta$. Using the constant reactivity $\rho$, from (21) we get

$$
\frac{d^{2} n}{d t^{2}}+\frac{d n}{d t}\left[\frac{\beta-\rho}{T}+\lambda\right]-n \frac{\lambda \rho}{T}=0 .
$$

It is not difficult to solve this equation by calculating the characteristic values $\kappa$ from the following characteristic equation:

$$
\kappa=\frac{1}{2}\left(\frac{\beta-\rho}{T}+\lambda\right)\left[ \pm \sqrt{1+4 \frac{\lambda T \rho}{(\beta+\lambda T-\rho)^{2}}}-1\right] .
$$

When $\rho<<\beta$ and $\lambda T<<\beta$ we find

$$
\kappa_{+}=\frac{\lambda \rho}{\beta}, \kappa_{-}=-\frac{\beta}{T},
$$

and when $\rho \sim \beta \gg \lambda T$ we have

$$
\kappa_{+}=\frac{\lambda \rho}{\beta-\rho}, \kappa_{-}=\frac{\rho-\beta}{T} .
$$

Equation (22) is similar to (17) and they become coinciding in the first order with respect to the small disturbances $\Delta \eta<<\eta_{0}$ if we set

$$
\rho=\Delta \eta .
$$

However, in the case of large disturbances $\Delta \eta \sim \eta_{0}$, these equations are qualitatively different. It is worth 
noting that Equations (17) and (22) are applicable when $\Delta \eta>\eta_{0}$ and $\rho>\beta$, respectively, while in the superstrong magnetic field the fraction of DN can become much larger than the value (12).

If in the classical case (22) the reactivity becomes greater then the fraction of $\mathrm{DN}, \rho>\beta$, then the root signs of $\kappa_{ \pm}$in (25) become opposite and the larger of them which is $\sim T^{-1}$ becomes positive. That is, the reactor starts to accelerate prompt neutrons, and thus gets out of control. In our case (17), we always have $\lambda>0$ and the root sign of $\kappa \sim T^{-1}$ in (18) can never change (19). Hence, in the new method of regulating a reactor, it shall never accelerate prompt neutrons (with significant $\kappa \sim T^{-1}$ ) but its power will always increase along with the value of $\kappa$ proportional to $\lambda$, i.e. inversely proportional to the lifetime of DN nuclear emitters.

\section{Conclusions}

Thus, if external impacts (e.g. superpstrong magnetic field) change the DN fraction, then the power of the reactor can be theoretically regulated. The reactor sets up initially subcritical, but it switches on and works under an external impact on the active zone. Such method will be much safer than the traditional one just because, even in the case of significant excitations, the reactor will neither be accelerated by instantaneous neutrons nor become "uncontrollable".

This work was partially supported by the \#2.1.1/2840 grant of The RF Education and Science Agency.

\section{References}

[1] M. Jung, F. Bosch, K. Beckert, et al. "First Observation of Bound-State Beta-Decay," Physics Review Letters, Vol. 69, No. 15, 1992, pp. 2164-2167.

[2] F. Bosch, T. Faestermann, J. Friese, et al. "Observation of Bound-State $\beta$-Decay of Fully Ionized ${ }^{187} \mathrm{Re}:{ }^{187} \mathrm{Re}-$ ${ }^{187}$ Os Cosmochronometry," Physics Review Letters, Vol. 77, No. 26, 1996, pp. 5190-5193.

[3] D. V. Filippov, "Increase in the Probability of Forbidden
Electron Beta Decays in a Superstrong Magnetic Field,' Nuclear Physics, Vol. 70, No. 12, 2007, pp. 2016-2024.

[4] D. V. Filippov, A. A. Rukhadze and L. I. Urutskoev, "Effects of Atomic Electrons on Nuclear Stability and Radioactive Decay," Ann. Fond. L. de Broglie, Vol. 29, No. 3, 2004, p. 1207.

[5] A. A. Rukhadze, D. V. Filippov and L. I. Urutskoev, "Effect of Beta Decay to Bound States in Ionized Atoms on the Fraction of Delayed Neutrons," Nuclear Physics A, Vol. 69, No. 5, 2006, pp. 792-795.

[6] Y. P. Gangrsky, F. F. Karpeshin, M. B. Trzhaskovskaya and Y. E. Penionzhkevich, "Effect of Beta-Electron Capture to a Bound State on Delayed-Neutron Emission from Fission Fragments," Nuclear Physics A, Vol. 71, No. 6, 2008, pp. 951-955.

[7] R. V. Meghreblian and D. K. Holmes, "Reactor Analysis," McGRAW-HILL, New York, 1960.

[8] J. N. Bahcall, "Theory of Bound-State Beta Decay," Physical Review, Vol. 124, No. 2, 1961, pp. 495-499.

[9] K. Takahashi and K. Yokoi, "Nuclear $\beta$-Decays of Highly Ionized Heavy Atoms in Stellar Interiors," Nuclear Physics A, Vol. 404, No. 3, 1983, pp. 578-598.

[10] K. Takahashi, et al. "Bound-State Beta Decay of Highly Ionized Atoms," Physical Review C, Vol. 36, No. 4, 1987, pp. 1522-1528.

[11] I. S. Batkin, "On the Decay of Bound States," Izv. Akad. Nauk SSSR, Ser. Fiz., Vol. 40, 1976, p. 1279.

[12] M. A. Preston, Physics of the Nucleus, Addison-Wesley, Reading, Massachusetts, 1962.

[13] Y. P. Gangrsky, B. Dalkhsuren and B. N. Markov, Nuclear-Fission Fragments, Energoatomizdat, Moscow, 1986.

[14] R. J. Elliott and R. Loudon, "Theory of the Absorption Edge in Semiconductors in a High Magnetic Field," Journal of Physics and Chemistry of Solids, Vol. 15, No. 3-4, 1960, pp. 196-207.

[15] B. B. Kadomtsev, "Heavy Atom in a Superstrong Magnetic Field," Soviet Physics-JETP, Vol. 31, 1970, p. 945.

[16] B. B. Kadomtsev and V. S. Kudryavtsev, "Atoms in a Superstrong Magnetic Field," JETP Letters, Vol. 13, No. 1, 1971, pp. 42-44. 